\title{
Protective effect of Barbaloin in a rat model of myocardial ischemia reperfusion injury through the regulation of the CNPY2-PERK pathway
}

\author{
YUE CUI, YONGQIANG WANG and GANG LIU \\ Department of Medicine, Tianjin Huanhu Hospital, Tianjin 300350, P.R. China
}

Received June 17, 2018; Accepted January 31, 2019

DOI: $10.3892 /$ ijmm.2019.4123

\begin{abstract}
Barbaloin (Bar) has a myocardial protective effect, but its mechanism of action is uncertain. The endoplasmic reticulum stress (ERS)-mediated apoptosis pathway serves an important role in the pathogenesis of myocardial ischemia-reperfusion injury (MIRI). Inhibiting ERS may significantly improve the progression of MIRI and serve a role in its prevention. Therefore, based on current knowledge of ERS-mediated cardiomyocyte apoptosis and the cardioprotective effect of Bar, the purpose of the present study was to further evaluate the myocardial protective effect and potential mechanisms of Bar pretreatment in MIRI. The present study established a MIR rat model and randomly divided these rats into four groups. Prior to myocardial ischemia, Bar $(20 \mathrm{mg} / \mathrm{kg})$ was administered to rats once daily for 1 week. Myocardial blood serum lactate dehydrogenase and creatine kinase were subsequently measured. A terminal deoxynucleotidyl transferase mediated dUTP nick end labeling assay was used to evaluate the myocardial protective effect of Bar pretreatment on MIRI. To assess whether the ERS signaling pathway was involved in the myocardial protection mechanism of Bar pretreatment, the expression levels of ERS-associated proteins, protein canopy homolog 2 (CNPY2), glucose regulatory protein 78, transcriptional activator 4, C/EBP-homologous protein (CHOP), PKR endoplasmic reticulum kinase (PERK), caspase-12 and caspase-3 were detected by western blot analysis, immunohistochemistry or reverse transcription-quantitative polymerase chain reaction. The results confirmed that Bar pretreatment significantly reduced the damage and the level of apoptosis caused by MIR. Bar pretreatment significantly inhibited the expression of ERS-associated proteins in cardiomyocytes. In addition, the immunohistochemistry results demonstrated that Bar pretreatment significantly inhibited the CNPY2-positive cell apoptosis ratio of cardiomyocytes. Therefore, the results
\end{abstract}

Correspondence to: Dr Yue Cui, Department of Medicine, Tianjin Huanhu Hospital, 6 Jizhao Road, Tianjin 300350, P.R. China

E-mail: cuiyue668@163.com

Key words: barbaloin, myocardial ischemia-reperfusion injury, endoplasmic reticulum stress, apoptosis of the current study suggested that CNPY2 is present in cardiomyocytes and participates in the development of MIRI by initiating the PERK-CHOP signaling pathway. Bar pretreatment may attenuate MIRI by inhibiting the CNPY2-PERK apoptotic pathway.

\section{Introduction}

Acute myocardial infarction is the primary cause of mortality in patients with coronary heart disease (1). The most commonly accepted and effective treatment is to restore the blood flow reperfusion as soon as possible (2). However, following the resumption of blood flow and reperfusion, ischemic myocardium aggravates cardiac function and causes additional structural destruction, leading to the further exacerbation of myocardial ischemic injury (MIR), known as myocardial ischemia-reperfusion injury (MIRI) (3). Identifying the key factors involved in MIRI and developing a rational intervention to reduce apoptosis following ischemia-reperfusion is a focus of clinical research.

The primary mechanisms of MIRI currently recognized include the release of excessive calcium, inflammatory factor release and cell apoptosis $(4,5)$. In recent years, increasing numbers of scholars have conducted in-depth studies regarding the mechanism of MIRI $(6,7)$. Previous studies have confirmed that the induction of apoptosis in MIR is the main form of acute MIRI (8-12). At present, three signal transduction pathways that regulate apoptosis have been identified, including the mitochondrial pathway, the death receptor pathway and the endoplasmic reticulum stress (ERS) pathway (13). Studies have demonstrated that the ERS-mediated apoptosis pathway serves an important role in the pathogenesis of MIRI $(14,15)$. When cardiomyocytes are affected by ischemia, the amount of unfolded protein in the lumen of the ER is significantly increased, which activates the ERS-associated apoptosis pathway $(16,17)$. Therefore, inhibiting ERS may significantly improve the progression of MIRI and serve a role in preventing MIRI.

Aloe is a succulent plant belonging to the family Liliaceae and has high economic value $(18,19)$. The role of aloe in immunity, anti-inflammation and anti-oxidation have been demonstrated previously (18), and has been used widely clinically (20). Barbaloin (Bar), the primary active ingredient in aloe has gained increasing attention (21). A recent study 
has demonstrated that Bar controls ventricular arrhythmia by regulating voltage-gated ion channels (22). Bar pretreatment inhibits myocardial oxidative stress by activating the AMP-activated protein kinase (AMPK) signaling pathway, thereby alleviating MIRI (23). However, to the best of our knowledge, no previous study has investigated whether Bar pretreatment inhibits myocardial apoptosis induced by MIR to achieve myocardial protection.

Therefore, based on the current knowledge on ERS-mediated cardiomyocyte apoptosis and the cardioprotective effects of Bar, the aim of the present study was to further evaluate the myocardial protective properties and potential mechanisms of action of Bar pretreatment in MIRI.

\section{Materials and methods}

Main reagents. Barbaloin (Bar, purity $>96 \%$ ) was purchased from Abcam (Cambridge, UK), and its solvent dimethyl sulfoxide (DMSO) was obtained from Merck KGaA (Sigma-Aldrich; Darmstadt, Germany).

Animals and drug treatment. A total of 96 healthy male Sprague-Dawley rats (SD, 8-10 weeks, 250-280 g) were selected and provided by the Tianjin Animal Center (Tianjin, China). All rats were housed in an environment of constant temperature $\left(22-25^{\circ} \mathrm{C}\right)$ and relative humidity (50-60\%) on a 12-h light/12-h dark cycle, and food and water were given ad libitum. All experimental protocols in the current study were performed in strict accordance with the institutional guidelines and approved by the Laboratory Animal Ethics Committee of Tianjin Huanhu Hospital (Tianjin, China) consistent with China Animal Care Committee.

All 96 healthy male rats were randomly divided into four groups: i) Sham operation + DMSO group (S group), after repeated intragastric administration of DMSO once a day for 1 week, the rats were threaded under the left anterior descending coronary artery (LAD) without ligation; ii) Bar alone group (Bar group), after repeated intragastric administration of Bar $(20 \mathrm{mg} / \mathrm{kg})$ once a day for 1 week, the rats were threaded under the LAD without ligation; iii) ischemia and reperfusion (I/R)+DMSO group, after repeated intragastric administration of DMSO once a day for 1 week, myocardial ischemia and reperfusion were induced for 30 and $120 \mathrm{~min}$, respectively; iv) I/R+Bar group, after repeated intragastric administration of Bar $(20 \mathrm{mg} / \mathrm{kg})$ once a day for 1 week, myocardial ischemia and reperfusion were induced for $30 \mathrm{~min}$ and $120 \mathrm{~min}$, respectively. The $\mathrm{S}$ and I/R groups were given equal volumes of DMSO.

Prior to the start of the experiment, SD rats were adapted to their environment for 1 week. As described previously, the rat model of MIR was prepared by ligation of the LAD (24). Briefly, rats were anesthetized with an intraperitoneal injection of $45 \mathrm{mg} / \mathrm{kg}$ pentobarbital sodium and mechanically ventilated using tracheal intubation. By identifying the origin $2 \mathrm{~mm}$ from the LAD, a 5/0 silk thread was used to surround the LAD. Following stabilization, transient ligation of LAD-induced ischemia was performed for $30 \mathrm{~min}$, which was followed by the loosening of the ligature line and reperfusion for $120 \mathrm{~min}$. Successful ligation of the LAD was confirmed by observing alterations to the ST segment on the electrocardiogram and color alterations in the ischemic areas of the heart (anterior wall and apex of the heart). After $120 \mathrm{~min}$ of reperfusion, $5 \mathrm{ml}$ of abdominal aortic blood was collected for serological examination. The weight of the experimental rats was 250-280 g. Subsequently, the heart was removed quickly and the blood remaining in the heart was cleared with cold physiological saline. The left ventricle was cut into two parts. One part was fixed in $4 \%$ paraformaldehyde for $24 \mathrm{~h}$ at room temperature, and the other was stored in a freezer at $-80^{\circ} \mathrm{C}$ for use in subsequent experimental analysis.

Determination of serum lactate dehydrogenase ( $L D H)$ and creatine kinase $(C K)$. After 120 min of reperfusion, abdominal aorta blood was collected and centrifuged at $900 \mathrm{x} \mathrm{g}$ for $10 \mathrm{~min}$ at $4^{\circ} \mathrm{C}$. The supernatant was subjected to serological analysis by spectrophotometry. The levels of LDH and CK were detected according to the manufacturer's protocols (Elabscience Biotechnology Co., Ltd., Wuhan, China).

Terminal deoxynucleotidyl transferase mediated dUTP nick end labeling (TUNEL) assay. At the end of the $120 \mathrm{~min}$ reperfusion, cardiomyocyte apoptosis was detected using a TUNEL assay. According to the manufacturer's protocol, the paraffin-embedded myocardial slices were stained using the in situ cell apoptosis detection kit (POD; Roche Diagnostics, Indianapolis, IN, USA). In the cardiac ischemic areas, five microscopic fields (x400) from each slice were randomly selected to determine the percentage of cells positively stained for apoptosis using a light microscope. The apoptosis rate was calculated as the number of apoptotic cells/total number of cells $\times 100 \%$.

Flow cytometric analysis. Cardiomyocyte apoptosis was analyzed by flow cytometry, as previously described (25). Double staining of fluorescein isothiocyanate (FITC)-conjugated Annexin V and propidium iodide (PI) was performed according to the manufacturer's protocol of the FITC-Annexin V and PI Apoptosis Detection kit (Beyotime Institute of Biotechnology, Haimen, China). The rats were anesthetized following $120 \mathrm{~min}$ of ischemia-reperfusion. The rat hearts were removed, washed with PBS and placed in glassware. The myocardial tissue was shredded, processed and washed with PBS, followed by centrifugation at $1,000 \mathrm{xg}$ for $5 \mathrm{~min}$ at $4^{\circ} \mathrm{C}$. The precipitate was collected and washed with PBS, and again centrifuged at $1,000 \mathrm{x}$ g for $5 \mathrm{~min}$ at $4^{\circ} \mathrm{C}$. The pellet was resuspended in a binding buffer containing $5 \mu \mathrm{l}$ of FITC-Annexin $\mathrm{V}$ and $10 \mu \mathrm{l}$ of PI staining solution. The solution was incubated for $15 \mathrm{~min}$ at room temperature in the dark. The mixture was filtered through a 300-mesh nylon net to remove impurities. Cellular fluorescence was analyzed using a flow cytometer (Becton, Dickinson and Company, Franklin, Lakes, NJ, USA). Apoptotic cells were measured as a percentage of the total number of cells, namely, the apoptotic index. The experiment was repeated three times.

Immunohistochemistry of protein canopy homolog 2 (CNPY2) in cardiomyocytes. The expression of CNPY2 in cardiomyocytes was detected using immunohistochemistry according to the manufacturer's protocol. Briefly, myocardial specimens were fixed in $4 \%$ formaldehyde for $24 \mathrm{~h}$ at 
room temperature, embedded in paraffin and sectioned into 4- $\mu \mathrm{m}$ thick slices. The slices were dewaxed and quenched in $3 \% \mathrm{H}_{2} \mathrm{O}_{2}$ at room temperature for $10 \mathrm{~min}$. Next, sections were incubated with anti-CNPY2 rabbit primary antibodies (1:50; cat. no. 14635-1-AP; ProteinTech Group, Inc., Chicago, IL, USA) for $2 \mathrm{~h}$ at $4^{\circ} \mathrm{C}$ followed by incubation with horseradish peroxidase (HRP)-conjugated Affinipure goat anti-rabbit immunoglobulin (Ig)G antibodies (1:100; cat. no. SA00001-2; ProteinTech Group, Inc.) at $4^{\circ} \mathrm{C}$ for $1 \mathrm{~h}$. Thereafter, sections were incubated in 3,3'-diamino benzidine for $15 \mathrm{~min}$ at room temperature for color rendering. The CNPY2-positive cells were observed at a $\mathrm{x} 400$ magnification under a light microscope. Brown staining indicated the presence of positive expression. Five areas of each sample from all experimental groups were randomly captured using the camera (Leica DM6000; Leica Microsystems GmbH, Wetzlar, Germany) and analyzed using ImagePro-Plus 6.0 software (Media Cybernetics, Inc., Rockville, MD, USA).

Extraction of myocardial total cell lysate and ER. After $2 \mathrm{~h}$ of reperfusion, left ventricular specimens from I/R injury were collected for western blot analysis. Protein isolation and western blotting were performed as previously described (26). The left ventricle specimens were lysed with $100 \mu 1$ of ice-cold RIPA lysis buffer (Beyotime Institute of Biotechnology) supplemented with $1 \mu \mathrm{l}$ of $100 \mathrm{mM}$ phenylmethylsulfonyl fluoride (Beyotime Institute of Biotechnology) for $30 \mathrm{~min}$. Tissue homogenates were centrifuged at $12,000 \mathrm{x}$ g for $30 \mathrm{~min}$ at $4^{\circ} \mathrm{C}$ and the total cell lysate was collected. Once the left ventricular samples were fully lysed, the tissue homogenate was centrifuged $\left(4^{\circ} \mathrm{C}, 800 \mathrm{x} \mathrm{g}\right)$ for $10 \mathrm{~min}$, and the new supernatant was centrifuged $\left(4^{\circ} \mathrm{C}, 10,000 \mathrm{x} \mathrm{g}\right)$ for $20 \mathrm{~min}$; the new supernatant was collected again for centrifugation $\left(4^{\circ} \mathrm{C}, 100,000 \mathrm{xg}\right)$ for $1 \mathrm{~h}$, the pellet of which was the ER.

Western blot analysis. The ER was suspended with a lysis solution containing $1 \%$ Triton X-100. The protein concentration of supernatants was quantified using the Bradford method. Protein samples (20 $\mu \mathrm{g} /$ lane) were separated on $10 \%$ SDS-PAGE gels, transferred to polyvinylidene difluoride membranes and blocked with $5 \%$ skim milk for $1 \mathrm{~h}$ at $4^{\circ} \mathrm{C}$. The membranes were incubated with primary antibodies at $4^{\circ} \mathrm{C}$ overnight: Phosphorylated-PKR endoplasmic reticulum kinase (p-PERK; 1:1,000; BIOSS, Beijing, China), CNPY2 (1:50; cat. no. 14635-1-AP), PERK (1:1,000; cat. no. 20582-1-AP), Calnexin (1:400; cat. no. 10427-2-AP) and GAPDH (1:2,000; cat. no. 10494-1-AP; all from ProteinTech Group, Inc.), glucose regulatory protein 78 (GRP78; 1:3,000; cat. no. ab21685), caspase-12 (1:10,000; cat. no. ab62484), transcriptional activator 4 (ATF4; 1:5,000; cat. no. ab23760), C/EBP-homologous protein (CHOP; 1:2,000; cat. no. ab11419) and caspase-3 (1:1,000; cat. no. ab13847; all from Abcam). Following washing three times with TBS with Tween-20, the membranes were incubated with HRP-conjugated Affinipure goat anti-mouse (cat. no. SA00001-1) or anti-rabbit (cat. no. SA00001-2; both 1:2,000; ProteinTech Group, Inc.) IgG antibodies for $1 \mathrm{~h}$ at room temperature. The target bands were visualized using enhanced chemiluminescence (EMD Millipore, Billerica, MA, USA). The quantification of the blots was measured using ImageJ software v.1.51u (National Institutes of Health,
Bethesda, MD, USA). All band intensities were normalized to GAPDH or Calnexin, and expressed as a percentage of the control sample.

Reverse transcription-quantitative polymerase chain reaction (RT-qPCR) analysis. According to the manufacturer's protocol, the total RNA was extracted from myocardial samples using TRIzol reagent (Invitrogen; Thermo Fisher Scientific, Inc., Waltham, MA, USA). The ReverTra Ace-a kit (Fermentas; Thermo Fisher Scientific, Inc., Pittsburgh, PA, USA) was used to reverse transcribe the total RNA ( $4 \mathrm{~g})$ to cDNA. PCR primers used were as follows: CNPY2 forward, 5'-CAGATT GACCCTTCTACCCACCG-3' and reverse, 5'-ATGCCGCCA TCTTCCTTCCCCTC-3'; GRP78 forward, 5'-TTCACTACT CTTGACCCTGCATCCC-3' and reverse, 5'-TTTCCTGCT TGAGCCGCTCGTTC-3'; Calnexin forward, 5'-GGCTTT GGGTGGTCTACATTCTG-3' and reverse, 5'-CATCCTCCT CTGCTTTAGGCTTG-3'. Data were normalized to Calnexin gene expression and analyzed by the comparative quantification method $\left(2^{-\Delta \Delta \mathrm{Cq}}\right)(27)$.

Statistical analysis. All data are presented as the mean \pm standard deviation of at least three independent replicates. Multiple groups were compared by one-way analysis of variance followed by Tukey's post hoc test using SPSS 16.0 (SPSS, Inc., Chicago, IL, USA). Data are presented as the mean \pm standard error of the mean. $\mathrm{P}<0.05$ was considered to indicate a statistically significant difference.

\section{Results}

Effects of Bar pretreatment on the serum levels of $\mathrm{LDH}$ and $C K$ induced by MIRI. To investigate the myocardial damage caused by MRI, alterations in the concentrations of myocardial injury markers LDH and CK. Compared with the S group, the contents of $\mathrm{CK}$ and $\mathrm{LDH}$ in the I/R group increased significantly (Fig. 1). Compared with the I/R, the contents of $\mathrm{CK}$ and LDH in the I/R+Bar group decreased significantly (Fig. 1). In addition, no significant difference in the concentrations of $\mathrm{CK}$ and LDH between the $\mathrm{S}$ group and the Bar group were observed. The results suggested that the administration of Bar may inhibit the increase of myocardial injury markers and may exhibit a protective effect on cardiac function.

Effects of Bar pretreatment on cardiomyocyte apoptosis induced by MIRI. To determine whether Bar pretreatment exhibited a protective effect on the myocardium, a TUNEL assay and flow cytometry were used to detect cardiomyocyte apoptosis. The results of the TUNEL assay demonstrated that the apoptosis rate of the I/R group was significantly higher compared with that of the $\mathrm{S}$ group. The proportion of apoptosis in the I/R+Bar group was significantly lower compared with that in the I/R group (Fig. 2A and B). Consistent with the results of the TUNEL assay, the flow cytometry results revealed that the apoptosis rate in the I/R group was significantly higher compared with that of the $\mathrm{S}$ group, and the percentage of cardiomyocyte apoptosis in the I/R group was significantly decreased following treatment with Bar (Fig. 3). There were no significant differences in apoptotic cells between the $\mathrm{S}$ group and the Bar group. The aforementioned 

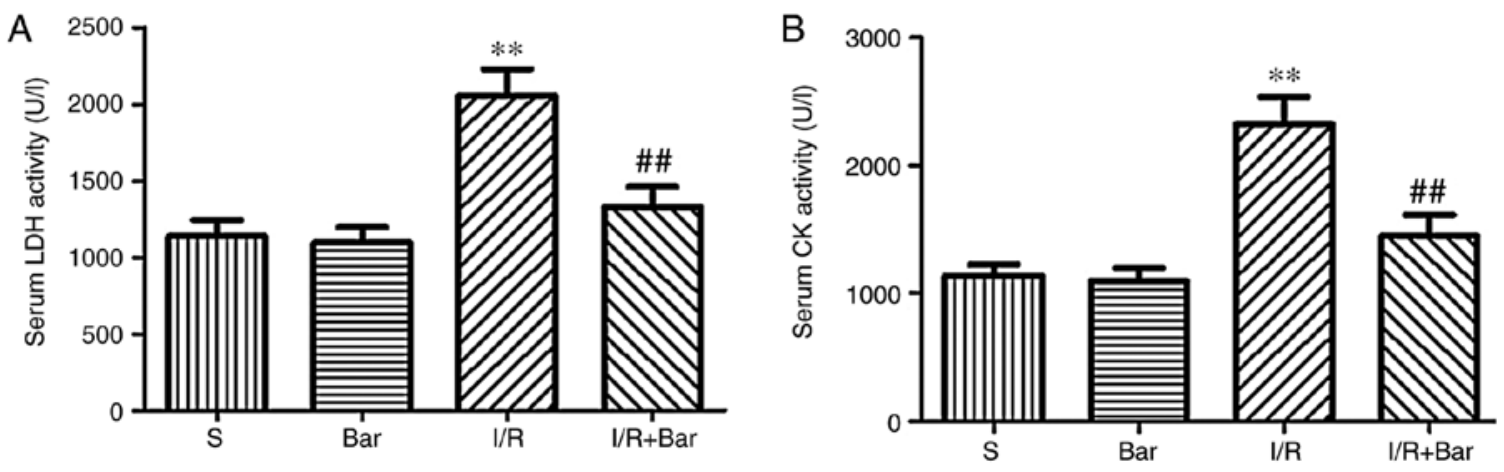

Figure 1. Effects of Bar pretreatment on the serum levels of LDH and CK following myocardial ischemia reperfusion injury. Serum levels (A) of LDH and (B) $\mathrm{CK} ; \mathrm{n}=6$. Data are presented as the mean \pm standard error of the mean. ${ }^{* *} \mathrm{P}<0.01$ compared with the $\mathrm{S}$ group; ${ }^{\# \#} \mathrm{P}<0.01$ compared with the $\mathrm{I} / \mathrm{R}$ group. Bar, barbaloin; LDH, lactate dehydrogenase; CK, creatine kinase; S, Sham operation + DMSO; I/R, ischemia and reperfusion.
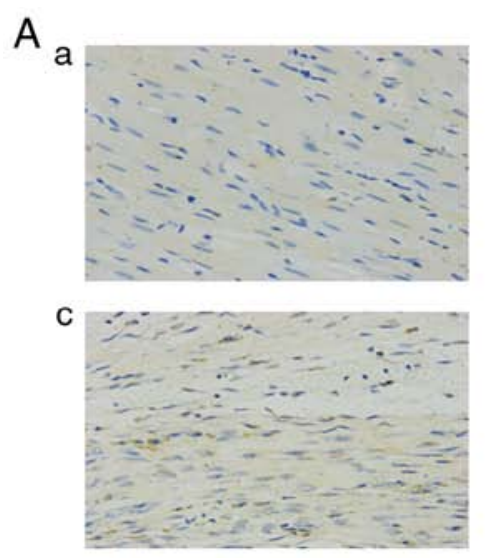

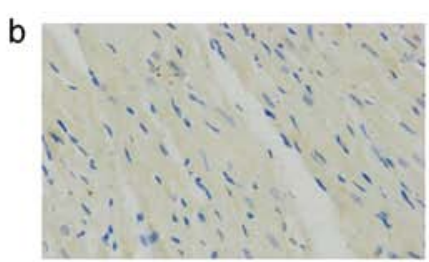

d

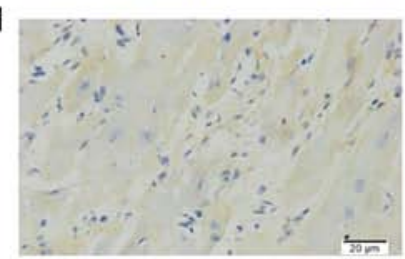

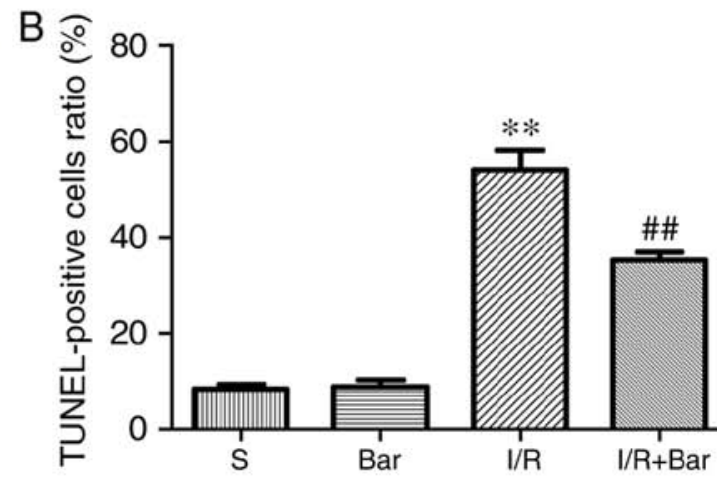

Figure 2. Effects of Bar pretreatment on myocardial apoptosis induced by myocardial ischemia reperfusion injury. (A) TUNEL staining was performed on the slices from myocardial cell specimens. (a) $\mathrm{S}$ group; (b) Bar group; (c) I/R group; (d) I/R+Bar group. (B) Ratio of positive cells in each group. Original magnification, $x 400 ; n=6$. Data are presented as the mean \pm standard error of the mean. ${ }^{* *} \mathrm{P}<0.01$ compared with the $\mathrm{S}$ group; ${ }^{\# \#} \mathrm{P}<0.01$ compared with the I/R group. Bar, barbaloin; S, Sham operation + DMSO; I/R, ischemia and reperfusion.

A
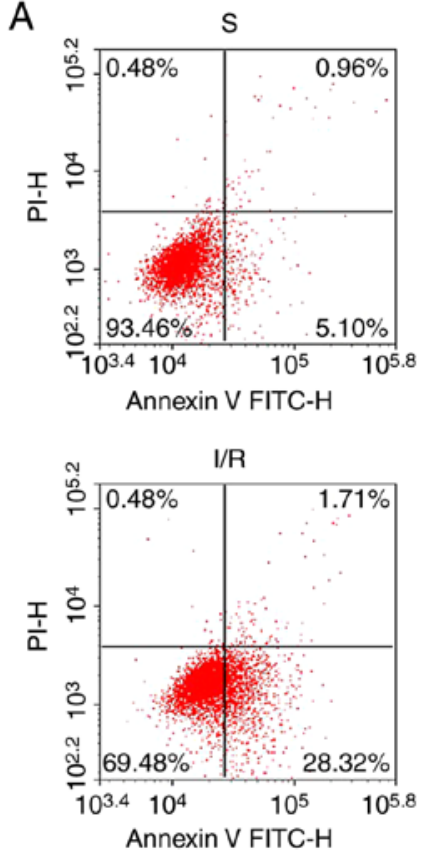
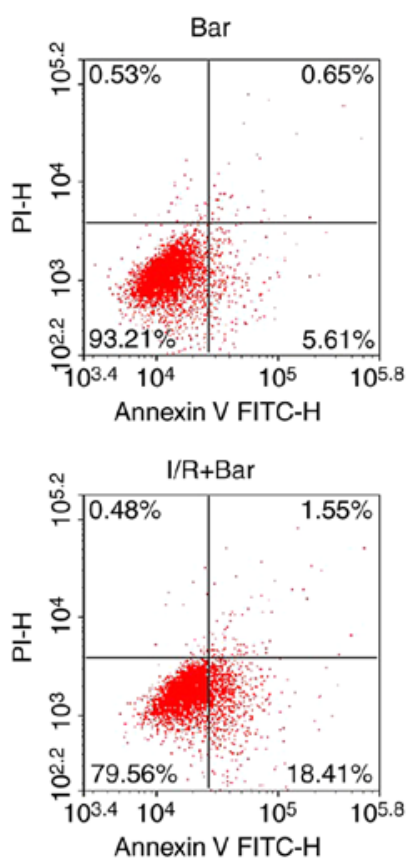

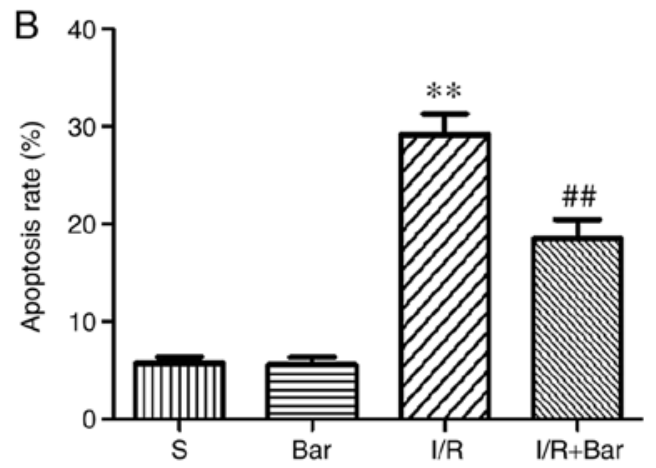

Figure 3. Effects of Bar pretreatment on myocardial apoptosis induced by myocardial ischemia reperfusion injury. (A) Flow cytometric analysis was performed on the slices from myocardial cell specimens. (B) Ratio of positive cells; $n=6$. Data are presented as the mean \pm standard error of the mean. ${ }^{* * *} P<0.01$ compared with the S group; ${ }^{\# \#} \mathrm{P}<0.01$ compared with the I/R group. Bar, barbaloin; S, Sham operation + DMSO; I/R, ischemia and reperfusion; PI, propidium iodide; FITC, fluorescein isothiocyanate. 
A

$\begin{array}{llll}S & \text { Bar } \quad \text { I/R } \quad \text { I/R+Bar }\end{array}$

p-PERK

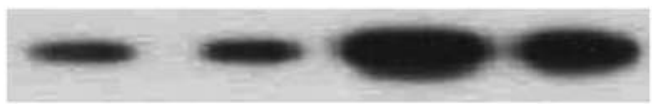

PERK

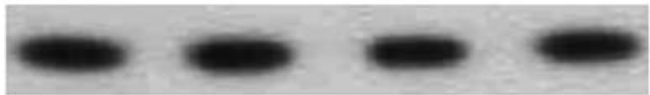

ATF4

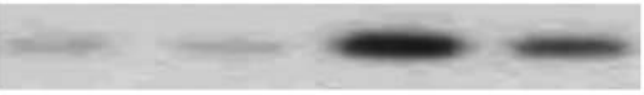

CHOP

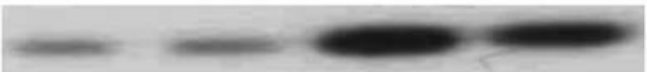

Caspase-12

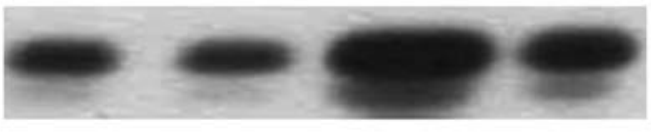

Caspase-3

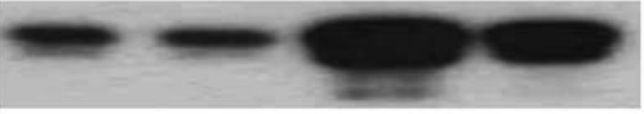

GAPDH

B a
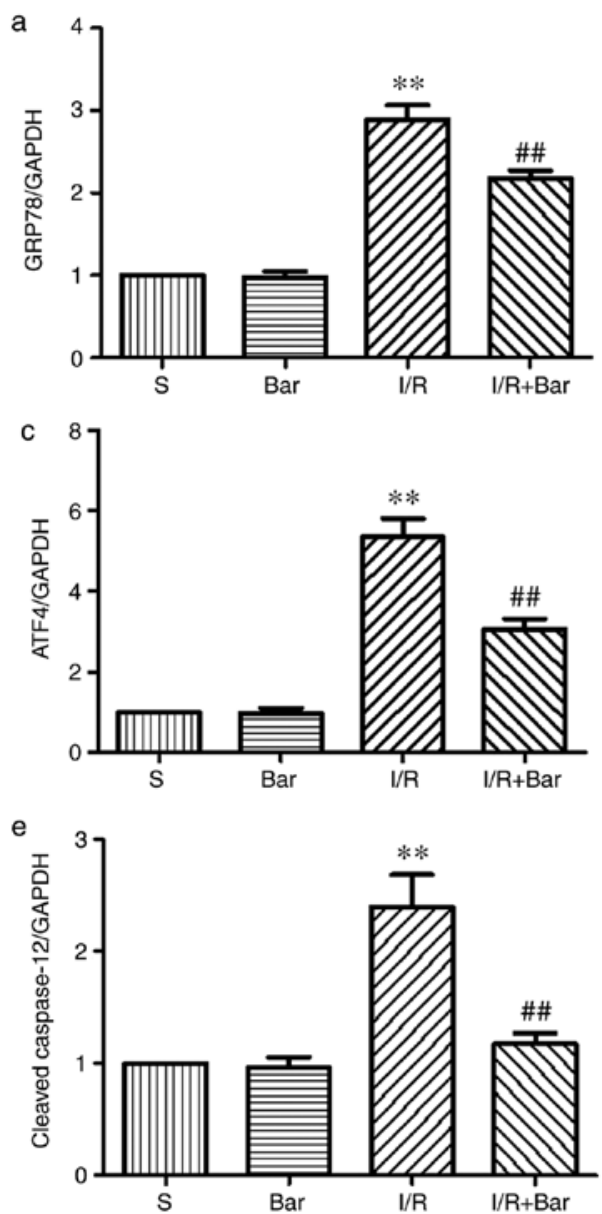
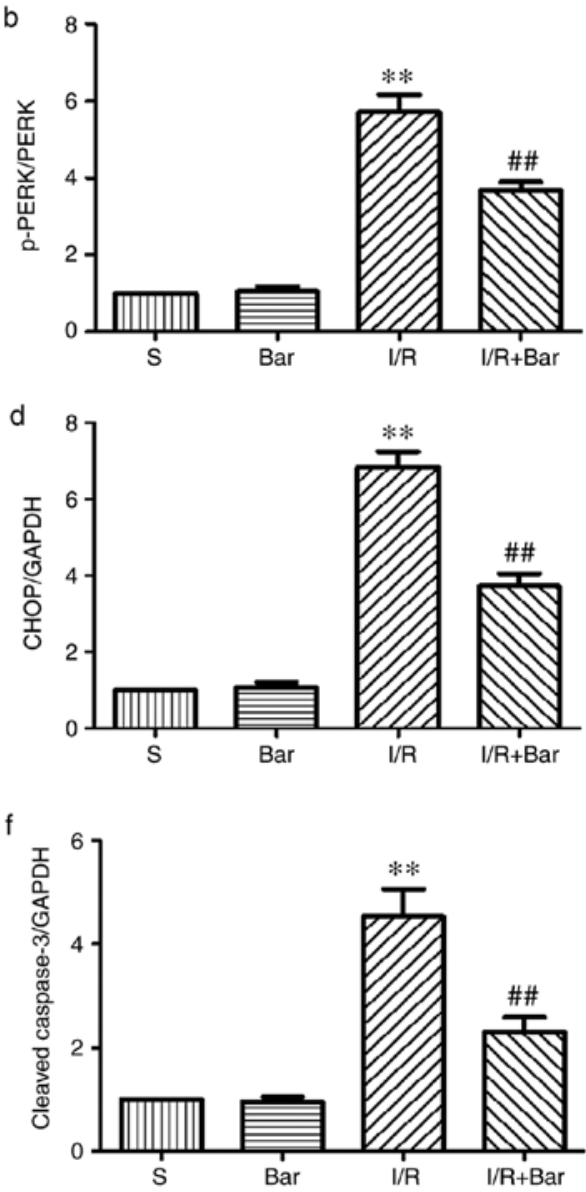

Figure 4. Effects of Bar pretreatment on the ERS-associated apoptosis pathway following myocardial ischemia reperfusion injury. (A) Representative western blot analysis and (B) quantification of the ERS-associated proteins (a) GRP78, (b) p-PERK, (c) ATF4, (d) CHOP, (e) caspase-12 and (f) caspase-3 from myocardial cell specimens. Expression of GAPDH was used as the loading control. The results were normalized to the percentage of GAPDH expression; $\mathrm{n}=6$. Data are presented as the mean \pm standard error of the mean. ${ }^{* *} \mathrm{P}<0.01$ compared with the $\mathrm{S}$ group; ${ }^{\# \#} \mathrm{P}<0.01$ compared with the $\mathrm{I} / \mathrm{R}$ group. Bar, barbaloin; $\mathrm{S}$, Sham operation + DMSO; I/R, ischemia and reperfusion; ERS, endoplasmic reticulum stress; GRP78, glucose regulatory protein 78; p-, phosphorylated; PERK, PKR endoplasmic reticulum kinase; ATF4, transcriptional activator 4; CHOP, C/EBP-homologous protein. 
A

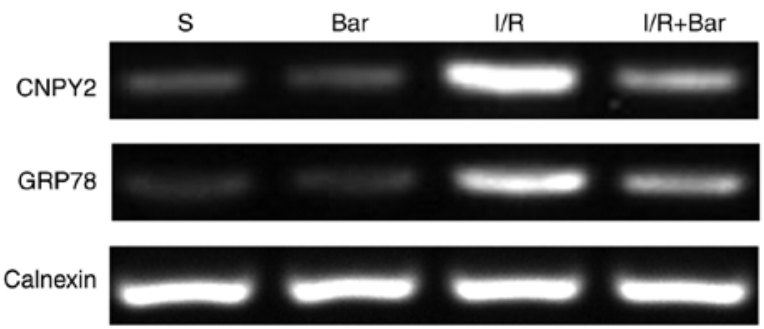

B a

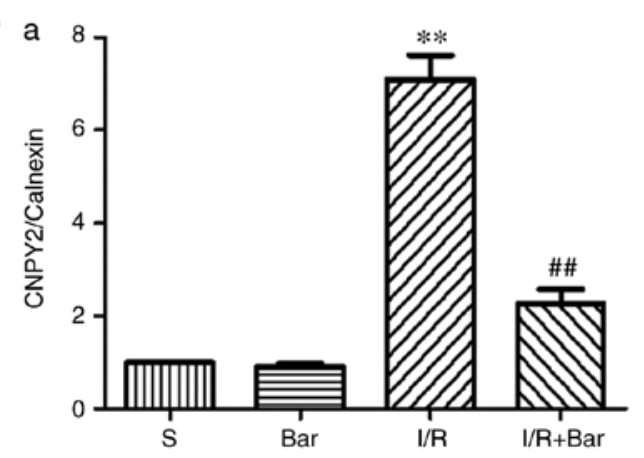

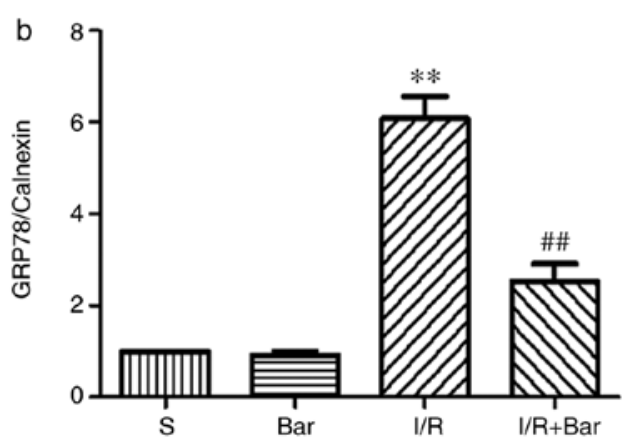

Figure 5. Effects of Bar pretreatment on the expression level of CNPY2 and GRP78 mRNA on the ER of cardiomyocytes following myocardial ischemia reperfusion injury. The mRNA expression of (B-a) CNPY2 and (B-b) GRP78 on ER was detected by reverse transcription-quantitative polymerase chain reaction (A). Expression of calnexin was used as the loading control. The results were normalized to the percentage of calnexin expression; $n=6$. Data are presented as the mean \pm standard error of the mean. ${ }^{* *} \mathrm{P}<0.01$ compared with the $\mathrm{S}$ group; ${ }^{\# \#} \mathrm{P}<0.01$ compared with the $\mathrm{I} / \mathrm{R}$ group. Bar, barbaloin; $\mathrm{S}, \mathrm{Sham}$ operation + DMSO; I/R, ischemia and reperfusion.
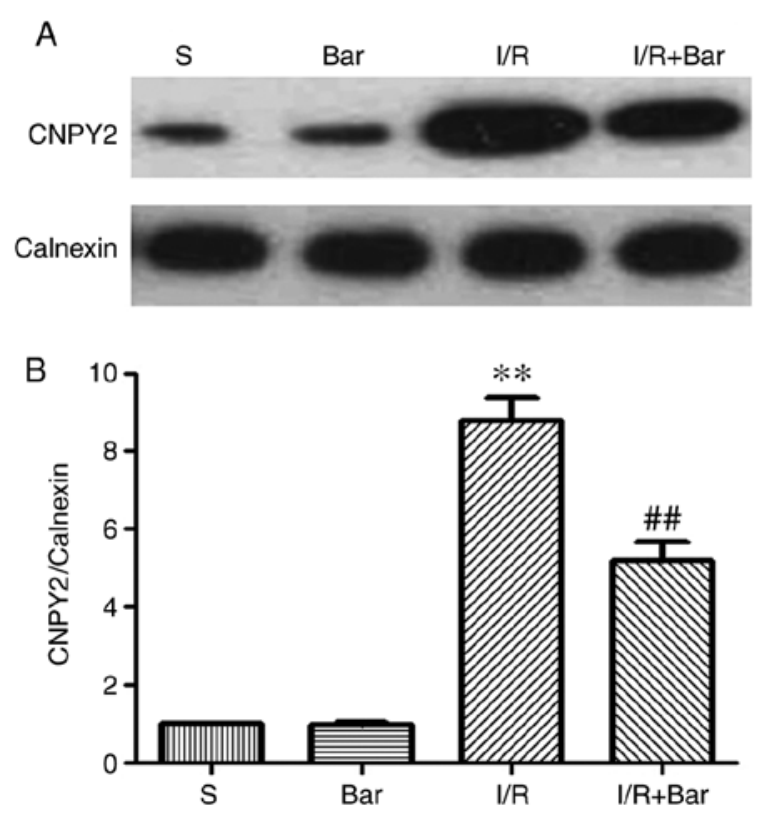

Figure 6. Effects of Bar pretreatment on CNPY2 induced by myocardial ischemia reperfusion injury. (A) Western blot analysis was used to measure the protein expression level of (B) CNPY2 on ER in cardiomyocytes. Expression of calnexin was used as the loading control. The results were normalized to the percentage of calnexin expression; $n=6$. Data are presented as the mean \pm standard error of the mean. ${ }^{* *} \mathrm{P}<0.01$ compared with the $\mathrm{S}$ group; ${ }^{\# \#} \mathrm{P}<0.01$ compared with the I/R group. Bar, barbaloin; S, Sham operation + DMSO; $\mathrm{I} / \mathrm{R}$, ischemia and reperfusion; CNPY2, protein canopy homolog 2 .

results suggested that Bar pretreatment significantly inhibited cardiomyocyte apoptosis.

Effects of Bar pretreatment on ERS induced by MIRI. To further demonstrate whether Bar pretreatment was able to protect the myocardium by inhibiting ERS, the expression of cytoplasmic ERS markers GRP78 and caspase-12 were analyzed (Fig. 4). Compared with the $\mathrm{S}$ group, the expression of GRP78 and cleaved caspase-12 protein in I/R group increased significantly. However, compared with the I/R group, the expression of GRP78 and cleaved caspase-12 protein in the I/R+Bar group was significantly decreased (Fig. 4B-a and -e). Furthermore, to verify the effect of Bar pretreatment on ERS, the expression levels of GRP78 mRNA on ERS of cardiomyocytes was detected. The mRNA expression of GRP78 was significantly higher in the $\mathrm{I} / \mathrm{R}$ group compared with that in the $\mathrm{S}$ group. Compared with the I/R group, the mRNA expression of GRP78 in the I/R+Bar group was significantly decreased (Fig. 5A and B-b). The results suggested that Bar pretreatment protected the myocardium by inhibiting ERS.

Effects of Bar pretreatment on CNPY2 induced by MIRI. To verify whether Bar affects the expression of CNPY2, mRNA and protein expression levels of CNPY2 on the ER of cardiomyocytes were detected (Figs. 5 and 6). Compared with the $\mathrm{S}$ group, the protein expression level of CNPY2 in the I/R group was significantly higher. Compared with the I/R group, the expression level of cardiomyocyte CNPY2 protein in the $\mathrm{I} / \mathrm{R}+\mathrm{Bar}$ group was significantly decreased (Fig. 6A and B). Compared with the $\mathrm{S}$ group, the mRNA level of CNPY2 was significantly increased in the I/R group. Compared with the $\mathrm{I} / \mathrm{R}$ group, mRNA expression of CNPY2 in the $\mathrm{I} / \mathrm{R}+\mathrm{Bar}$ group decreased significantly, which was consistent with the western blotting results (Fig. 5A and B-a). The expression of CNPY2 in cardiomyocytes was detected by immunohistochemistry, as shown in Fig. 7. The expression of CNPY2 in the I/R group was significantly higher compared with that in the $\mathrm{S}$ group. Compared with the I/R group, the expression of CNPY2 decreased significantly following Bar pretreatment, which was 
A
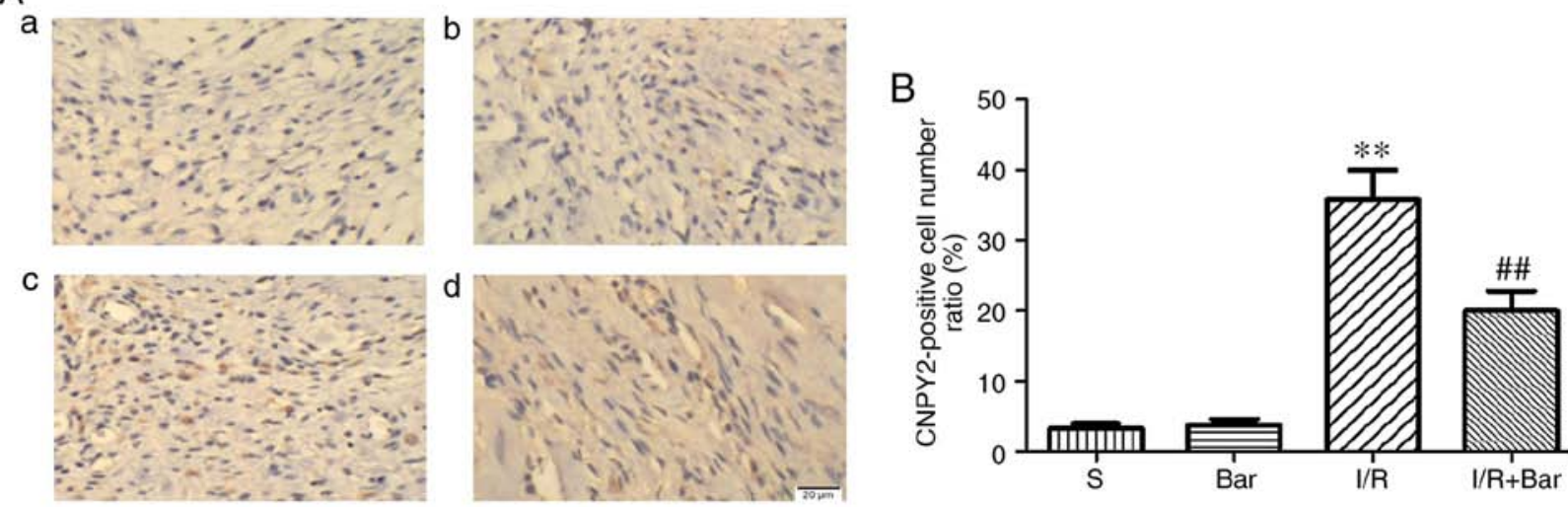

Figure 7. Effects of Bar pretreatment on CNPY2 expression levels in cardiomyocytes after myocardial ischemia reperfusion injury. (A) Immunohistochemical study of CNPY2 was performed on slices from myocardial cell specimens. (a) S group; (b) Bar group; (c) I/R group; (d) I/R+Bar group. (B) Ratio of positive cells in each group. Original magnification, $\mathrm{x} 400 ; \mathrm{n}=6$. Data are presented as the mean \pm standard error of the mean. ${ }^{* *} \mathrm{P}<0.01$ compared with the $\mathrm{S}$ group; ${ }^{\# \#} \mathrm{P}<0.01$ compared with the I/R group. Bar, barbaloin; S, Sham operation + DMSO; I/R, ischemia and reperfusion; CNPY2, protein canopy homolog 2.

consistent with western blot analysis and RT-qPCR results (Fig. 7A and B). The aforementioned results confirmed that CNPY2 is expressed in cardiomyocytes, which is consistent with previous studies $(28,29)$. Bar pretreatment may protect the myocardium from ischemia-reperfusion injury by inhibiting the CNPY2-associated signaling pathway.

Effects of Bar pretreatment on the ERS-associated apoptosis pathway following MIRI. To further examine the effect of Bar pretreatment on the ERS-associated apoptosis pathway following MIRI, the expression of ERS-associated apoptotic pathway proteins was detected. The western blot analysis results demonstrated no significant differences in the expression levels of p-PERK, PERK, CHOP, ATF4 or cleaved caspase-3 between the $\mathrm{S}$ and Bar alone groups (Fig. 4). However, the expression of p-PERK, PERK, ATF4, CHOP and cleaved caspase -3 proteins in the $\mathrm{I} / \mathrm{R}+\mathrm{Bar}$ group was significantly decreased compared with that of the I/R group (Fig. 4). These results indicated that Bar pretreatment protected myocardial cells from ischemia-reperfusion injury by inhibiting the PERK-CHOP mediated apoptotic signal pathway.

\section{Discussion}

Ischemic heart disease is a serious health problem worldwide and its incidence is increasing $(30,31)$. Rapidly restoring myocardial blood flow and relieving tissue ischemia is the only effective treatment for ischemic heart disease $(32,33)$. However, an important problem in restoring blood perfusion is MIRI (33). Following an in depth study of the mechanism of ischemic precondition for myocardial protection, it may be suggested that other physical stimuli or certain drug preparations also lead to preconditioning, thereby enhancing the ability of cells to resist injury (30). Therefore, it is important to understand the pathogenesis of MIRI and actively identify effective therapeutic targets to prevent MIRI. In recent years, drug pretreatment has become an interesting topic in the prevention and treatment of MIR.

MIRI is a complex pathophysiological process with the understanding of its pathogenesis and signal transduction remaining unclear. It is considered that cardiomyocyte apoptosis, free radical production and intracellular calcium overload are important pathogenic mechanisms of MIRI $(8,9)$. Apoptosis is a process of programmed cell death, which is involved in a variety of physiological and pathological processes (34). Growing evidence from basic and clinical experiments has demonstrated that cardiomyocyte apoptosis is associated with the occurrence and development of ischemic cardiomyopathy $(12,34)$. Apoptosis is an important cause of myocardial cell injury and cardiac function decline following MIR $(12,35)$. The results of the present study demonstrated that MIRI increased the levels of myocardial injury markers $\mathrm{CK}$ and $\mathrm{LDH}$, and increased the proportion of myocardial cells undergoing apoptosis. This indicates that MIR may cause myocardial cell apoptosis and exacerbate the damage to cardiac function.

The classical apoptotic pathways in cells primarily include the death receptor activation pathway and the mitochondrial damage apoptotic pathway $(36,37)$. The apoptosis pathway mediated by ERS is a more recently discovered pathway of apoptosis (9). The ER is the site of protein folding, calcium homeostasis and lipid biosynthesis. It is an important organelle for determining the survival of cells (38). The dynamic balance of the intracellular environment serves a decisive role in the maintenance of the physiological function of the ER. In a variety of pathological conditions, the homeostasis of the ER is destroyed, and its function is disorganized, which results in the accumulation of a large number of misfolded and unfolded proteins. This process is called ERS (39). At this point, the cell starts a highly conservative unfolded protein response (UPR) to improve the ability of the ER to process and refold the proteins to maintain the homeostasis of the cells. The UPR process is primarily mediated by three ER transmembrane proteins: PERK, inositol dependent enzyme 1 (IRE1) and ATF6. The binding of these three sentinel proteins to the ER chaperone GRP78 is inactivated (39). However, when persistent, intense ERS occurs, the overexpression of GRP78 is induced, which activates the expression of PERK, IRE1 and ATF6 and triggers the CHOP and caspase-12 apoptosis signaling pathway $(39,40)$. This experiment showed that the expression of ERS marker proteins GRP78 and caspase-12 increased significantly after 
ischemia-reperfusion, demonstrating that ERS was involved in the occurrence of MIRI.

Aloe is a kind of plant belonging to Liliaceae $(18,19)$. It is a traditional Chinese medicine used to treat wounds and diarrhea. It has attracted the attention of many scholars because of its pharmacological properties (18). It has been reported that aloe can alleviate neuronal damage and protect the spinal cord from ischemia-reperfusion injury (41). Aloe has an effective neuroprotective effect on sciatic nerve ischemia-reperfusion injury (42). Aloe may serve a protective role in cerebral ischemia by inhibiting neuronal apoptosis (43). The gavaging of aloe has been demonstrated to protect kidney and lung tissues from oxidative damage caused by ischemia-reperfusion (44). Bar is the main active ingredient of aloe and has received increasingly more attention (21). A recent study demonstrated that Bar controlled ventricular arrhythmia by regulating voltage-gated ion channels (22). Bar pretreatment inhibits myocardial oxidative stress by activating the AMPK signaling pathway, thereby alleviating MIRI (23). However, to the best of our knowledge, no previous studies have demonstrated that Bar pretreatment inhibits myocardial apoptosis induced by MIR to achieve myocardial protection. In the present study, following pretreatment with Bar, the concentration of myocardial injury markers CK and LDH decreased significantly, and the apoptosis of myocardial cells was significantly reduced. The experimental results suggested that the expression of ERS marker proteins GRP78 and caspase-12 were markedly decreased following the application of Bar. The aforementioned experimental results suggest that Bar pretreatment exhibited a protective effect on MIRI and ERS participated in this protection mechanism.

ER is a membranous organelle. It is a specific site for monitoring protein synthesis, folding, modification and aggregation (45). These proteins serve an important role in regulating cell activity in the ER, golgi apparatus and the cell membrane. Correct protein folding is key to cell survival (39). Therefore, UPR is an important protein reaction for the function and maintenance of normal physiological activities of the ER $(37,39)$. The ER has a complex monitoring system that ensures the correct folding, modification and assembly of proteins through a variety of regulatory mechanisms. Ischemia-reperfusion injury disrupts the homeostasis of the ER, causing unfolded or misfolded proteins, thereby activating UPR and promoting cell survival $(37,39)$. The UPR pathway is continuously regulated under normal and stress conditions. However, how UPR sensors are triggered by molecules remains unclear. A previous study reported that the deletion of CNPY2 inhibited the PERK-CHOP signaling pathway and protected mice from UPR-induced liver injury (46). The PERK-CNPY2 axis enhanced ER stress-induced cell death in liver disease (47). Recent studies have demonstrated that CNPY2 is present in cardiomyocytes, and have confirmed that CNPY2 attenuates the shift from compensatory hypertrophic response to ventricular dilatation and heart failure $(28,29)$. CNPY2 serves a key role in ERS and is an important trigger factor for the PERK-CHOP signaling pathway $(46,47)$. Therefore, we hypothesized that CNPY2 may also initiate the PERK-CHOP signaling pathway and participate in the ERS induced by MIRI. The results of the current study revealed that CNPY2 was expressed in rat cardiomyocytes, and may activate the PERK-CHOP pathway to trigger the ERS apoptotic pathway-associated proteins CHOP and caspase-3, eventually leading to cardiomyocyte apoptosis.

In conclusion, the results of the present study suggest that CNPY2 is present in cardiomyocytes and may participate in the development of MIRI by initiating the PERK-CHOP signaling pathway. Bar pretreatment reduced MIRI by inhibiting the CNPY2-PERK apoptotic pathway. These findings provide novel insights into the pathogenesis of MIRI and demonstrate the potential mechanisms by which Bar treats myocardial infarction, which is important for developing novel strategies for the prevention and treatment of myocardial infarction. However, the pathogenesis of myocardial infarction is complex, and there are other signaling pathways involved in cardiomyocyte apoptosis. To further clarify the therapeutic mechanism of Bar for myocardial infarction, further studies are required.

\section{Acknowledgements}

Not applicable.

\section{Funding}

The present study was supported by the Foundation of Tianjin Health and Family Planning Commission (grant no. 14KG101).

\section{Availability of data and materials}

All data generated or analyzed during the current study are included in this published article.

\section{Authors' contributions}

YC and GL conceived and designed the study. YC conducted the experiments and wrote the manuscript. YC and YW analyzed the data. YC and GL revised the manuscript. All the authors read and approved the final manuscript.

\section{Ethics approval and consent to participate}

All experimental protocols in the current study were performed in strict accordance with the institutional guidelines and approved by the Laboratory Animal Ethics Committee of Tianjin Huanhu Hospital (Tianjin, China) consistent with China Animal Care Committee.

\section{Patient consent for publication}

Not applicable.

\section{Competing interests}

The authors declare that they have no competing interests.

\section{References}

1. Puaschitz NG, Assmus J, Strand E, Karlsson T, Vinknes KJ, Lysne V, Drevon CA, Tell GS, Dierkes J and Nygård O: Adherence to the Healthy Nordic Food Index and the incidence of acute myocardial infarction and mortality among patients with stable angina pectoris. J Hum Nutr Diet 32: 86-97, 2019. 
2. Prondzinsky R, Lemm H, Geppert A, Buerke M, Russ M and Werdan K: Infarct-related cardiogenic shock: Prognosis and treatment. Med Klin Intensivmed Notfmed 113: 267-276, 2018 (In German)

3. Zhou H, Ma Q, Zhu P, Ren J, Reiter RJ and Chen Y: Protective role of melatonin in cardiac ischemia-reperfusion injury: From pathogenesis to targeted therapy. J Pineal Res Epub: Feb 8, 2018 (Epub ahead of print). doi: 10.1111/jpi.12471.

4. Wu MY, Yiang GT, Liao WT, Tsai AP, Cheng YL, Cheng PW, $\mathrm{Li} \mathrm{CY}$ and Li CJ: Current mechanistic concepts in ischemia and reperfusion injury. Cell Physiol Biochem 46: 1650-1667, 2018.

5. Muntean DM, Sturza A, Dănilă MD, Borza C, Duicu OM and Mornos C: The role of mitochondrial reactive oxygen species in cardiovascular injury and protective strategies. Oxid Med Cell Longev 2016: 8254942, 2016.

6. Ye G,Fu Q, Jiang L and Li Z: Vascular smooth muscle cells activate $\mathrm{PI} 3 \mathrm{~K} / \mathrm{Akt}$ pathway to attenuate myocardial ischemia/reperfusion-induced apoptosis and autophagy by secreting bFGF. Biomed Pharmacother 107: 1779-1785, 2018.

7. Zhang W, Zhang Y, Ding K, Zhang H, Zhao Q, Liu Z and Xu Y: Involvement of JNK1/2-NF- $\mathrm{BBp} 65$ in the regulation of HMGB2 in myocardial ischemia/reperfusion-induced apoptosis in human AC16 cardiomyocytes. Biomed Pharmacother 106: 1063-1071, 2018.

8. Xia P, Liu Y and Cheng Z: Signaling pathways in cardiac myocyte apoptosis. Biomed Res Int 2016: 9583268, 2016.

9. Haunstetter A and Izumo S: Apoptosis: Basic mechanisms and implications for cardiovascular disease. Circul Res 82: 1111-1129, 1998.

10. Baines CP and Molkentin JD: STRESS signaling pathways that modulate cardiac myocyte apoptosis. J Mol Cell Cardiol 38 47-62, 2005.

11. Bernecker OY, Huq F, Heist EK, Podesser BK and Hajjar RJ: Apoptosis in heart failure and the senescent heart. Cardiovasc Toxicol 3: 183-190, 2003

12. Mattson MP and Kroemer G: Mitochondria in cell death: Novel targets for neuroprotection and cardioprotection. Trends Mol Med 9: 196-205, 2003.

13. Rao RV, Ellerby HM and Bredesen DE: Coupling endoplasmic reticulum stress to the cell death program. Cell Death Differ 11: 372-380, 2004

14. Minamino T and Kitakaze M: ER stress in cardiovascular disease. J Mol Cell Cardiol 48: 1105-1110, 2010.

15. Zhang C, Tang Y, Li Y, Xie L, Zhuang W, Liu J and Gong J: Unfolded protein response plays a critical role in heart damage after myocardial ischemia/reperfusion in rats. PLoS One 12 e0179042, 2017.

16. Kalogeris T, Baines CP, Krenz M and Korthuis RJ: Ischemia/reperfusion. Compr Physiol 7: 113-170, 2016.

17. Xu C, Bailly-Maitre B and Reed JC: Endoplasmic reticulum stress: Cell life and death decisions. J Clin Invest 115: 2656-2664, 2005.

18. Singab AN, El-Hefnawy HM, Esmat A, Gad HA and Nazeam JA: A systemic review on Aloe arborescens pharmacological profile: Biological activities and pilot clinical trials. Phytother Res 29: 1858-1867, 2015

19. Anuszewska EL: Mechanisms of therapeutic action of aloe. Wiad Lek 68: 168-172, 2015 (In Polish).

20. Akaberi M, Sobhani Z, Javadi B, Sahebkar A and Emami SA Therapeutic effects of Aloe spp. in traditional and modern medicine: A review. Biomed Pharmacother 84: 759-772, 2016.

21. Patel DK, Patel K and Tahilyani V: Barbaloin: A concise report of its pharmacological and analytical aspects. Asian Pac J Trop Biomed 2: 835-838, 2012

22. Cao ZZ, Tian YJ, Hao J, Zhang PH, Liu ZP, Jiang WZ, Zeng ML, Zhang PP and Ma JH: Barbaloin inhibits ventricular arrhythmias in rabbits by modulating voltage-gated ion channels. Acta Pharmacol Sin 39: 357-370, 2018.

23. Zhang P, Liu X, Huang G, Bai C, Zhang Z and Li H: Barbaloin pretreatment attenuates myocardial ischemia-reperfusion injury via activation of AMPK. Biochem Biophys Res Commun 490: 1215-1220, 2017.

24. Chimenti S, Carlo E, Masson S, Bai A and Latini R: Myocardial infarction: Animal models. Methods Mol Med 98: 217-226, 2004

25. Li F, Zheng X, Fan X, Zhai K, Tan Y, Kou J and Yu B: YiQiFuMai powder injection attenuates ischemia/reperfusion-induced myocardial apoptosis through AMPK activation. Rejuvenation Res 19: 495-508, 2016.
26. Qi X, Vallentin A, Churchill E and Mochly-Rosen D: DeltaPKC participates in the endoplasmic reticulum stress-induced response in cultured cardiac myocytes and ischemic heart. J Mol Cell Cardiol 43: 420-428, 2007.

27. Livak KJ and Schmittgen TD: Analysis of relative gene expression data using real-time quantitative PCR and the 2(-Delta Delta C(T)) method. Methods 25: 402-408, 2001.

28. Guo J, Mihic A, Wu J, Zhang Y, Singh K, Dhingra S, Weisel RD and Li RK: Canopy 2 attenuates the transition from compensatory hypertrophy to dilated heart failure in hypertrophic cardiomyopathy. Eur Heart J 36: 2530-2540, 2015.

29. Hatta K, Guo J, Ludke A, Dhingra S, Singh K, Huang ML, Weisel RD and Li RK: Expression of CNPY2 in mouse tissues: Quantification and localization. PLoS One 9: e111370, 2014.

30. Ding S, Fan Z, Lin C, Dai Q, Zhou J, Huang H, Xu Y and Zhong C: Therapeutic effects of ischemic-preconditioned exosomes in cardiovascular diseases. Adv Exp Med Biol 998: 271-281, 2017.

31. Yasuda S and Shimokawa H: Acute myocardial infarction: The enduring challenge for cardiac protection and survival. Circ J 73: 2000-2008, 2009.

32. Kalra S, Bhatt H and Kirtane AJ: Stenting in primary percutaneous coronary intervention for acute ST-segment elevation myocardial infarction. Methodist Debakey Cardiovasc J 14: $14-22,2018$

33. Piper HM, Kasseckert SA, Schlüter KD and Abdallah Y: Pathophysiology of myocardial reperfusion injury. Dtsch Med Wochenschr 133: 586-590, 2008

34. Crow MT, Mani K, Nam YJ and Kitsis RN: The mitochondrial death pathway and cardiac myocyte apoptosis. Circ Res 95: 957-970, 2004

35. Xu T, Ding W, Tariq MA, Wang Y, Wan Q, Li M and Wang J: Molecular mechanism and therapy application of necrosis during myocardial injury. J Cell Mol Med 22: 2547-2557, 2018.

36. Khosravi-Far R and Esposti MD: Death receptor signals to mitochondria. Cancer Biol Ther 3: 1051-1057, 2004.

37. Tabas I and Ron D: Integrating the mechanisms of apoptosis induced by endoplasmic reticulum stress. Nat Cell Biol 13: 184-190, 2011.

38. Gorman AM, Healy SJ, Jäger R and Samali A: Stress management at the ER: Regulators of ER stress-induced apoptosis. Pharmacol Ther 134: 306-316, 2012.

39. Iurlaro R and Muñoz-Pinedo C: Cell death induced by endoplasmic reticulum stress. FEBS J 283: 2640-2652, 2016.

40. Ferri KF and Kroemer G: Organelle-specific initiation of cell death pathways. Nat Cell Biol 3: E255-E263, 2001.

41. Yuksel Y, Guven M, Kaymaz B, Sehitoglu MH, Aras AB Akman T, Tosun M and Cosar M: Effects of aloe vera on spinal cord ischemia-reperfusion injury of rats. J Invest Surg 29: 389-398, 2016.

42. Guven M, Gölge UH, Aslan E, Sehitoglu MH, Aras AB, Akman T and Cosar M: The effect of aloe vera on ischemia-reperfusion injury of sciatic nerve in rats. Biomed Pharmacother 79: 201-207, 2016.

43. Lu ZQ, Deng YJ and Lu JX: Effect of aloe polysaccharide on caspase-3 expression following cerebral ischemia and reperfusion injury in rats. Mol Med Rep 6: 371-374, 2012

44. Sahin H, Yener AU, Karaboga I, Sehitoglu MH, Dogu T, Altinisik HB, Altinisik U and Simsek T: Protective effect of gel form of gastric gavage applicated aloe vera on ischemia reperfusion injury in renal and lung tissue. Cell Mol Biol (Noisy-le-grand) 63: 34-39, 2017.

45. Ron D and Walter P: Signal integration in the endoplasmic reticulum unfolded protein response. Nat Rev Mol Cell Biol 8: 519-529, 2007.

46. Hong F, Liu B, Wu BX, Morreall J, Roth B, Davies C, Sun S, Diehl JA and Li Z: CNPY2 is a key initiator of the PERK-CHOP pathway of the unfolded protein response. Nat Struct Mol Biol 24: 834-839, 2017.

47. Urra $\mathrm{H}$ and Hetz $\mathrm{C}$ : Fine-tuning PERK signaling to control cell fate under stress. Nat Struct Mol Biol 24: 789-790, 2017.

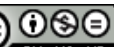

This work is licensed under a Creative Commons Attribution-NonCommercial-NoDerivatives 4.0 International (CC BY-NC-ND 4.0) License. 